\title{
A 3D NUMERICAL MODEL FOR COMPUTING NON-BREAKING WAVE FORCES ON SLENDER PILES
}

\author{
Weihua $\mathrm{Mo}^{1}$, Kai Irschik ${ }^{2}$, Hocine Oumeraci ${ }^{2}$, Philip L.-F. Liu ${ }^{1}$ \\ ${ }^{1}$ School of Civil and Environmental Engineering, Cornell University, Ithaca, NY, USA \\ ${ }^{2}$ Leichtweiß-Institute, Beethovenstr. 51a, 38106 Braunschweig, Germany
}

\begin{abstract}
In this paper we validate a numerical model for water-wave-body interaction by comparing the numerical results with laboratory data. The numerical model is based on the Euler's equation without considering the effects of energy dissipation. The Euler's equations are solved by a two-step projection finite volume scheme and the free surface displacements are tracked by the volume of fluid method. The numerical model is used to simulate solitary wave as well as periodic waves and their interaction with a vertical slender pile. A very good agreement between the experimental data and numerical results is observed for the time history of free surface displacement, fluid particle velocity, and dynamic pressure on the pile.
\end{abstract}

\section{Introduction}

Cylindrical piles are among the most commonly used structures in coastal and offshore engineering. In the nearshore region they are used for jetties or piers and in deepwater for offshore platforms (see Figure 1). In designing coastal and offshore structures, it is essential to accurately estimate wave forces acting on the piles. In the case of a slender pile, where the diameter of the pile $(D)$ is small in comparison with the design wave length $(\lambda)$, the Morison equation [1] represents a good approximation for calculating the wave forces. On the other hand, if the diameter of the pile is not sufficiently small, the presence of the pile will generate significant scattered waves and the wave forces can be accurately calculated only if the interaction between waves and cylinder is fully considered [2].



Figure 1: Slender cylindrical structures in coastal and offshore engineering
Regardless the size of the pile, information on wave forces can be obtained by means of experimental approaches. Even when the Morison equation approach is taken, the dependency of two coefficients, $C_{D}$ (drag coefficient) and $C_{M}$ (mass coefficient), on the wave conditions and the geometry of the pile needs to be determined empirically. Because of considerable costs and time required by laboratory experiments it is not very often feasible to perform extensive parameter studies (e.g., variation of water depth, diameter and inclination of piles, wave parameters, breaker type, and configuration of piles in a group). The alternative is to use numerical simulations as supplements to laboratory experiments. Moreover, an accurate numerical simulation might provide much more detailed insights into the physical processes that could hardly be achieved by experimental approach.

Modeling the wave-pile interaction faces many challenges. First of all, the flow of interest is a complex three-dimensional free surface flow with moving runup boundaries on the 
pile. For large incident waves, they might break in front of the pile and flow separation might occur on the lee side of the pile. Therefore, local, but strong, turbulence in the vicinity of the pile and near the free surface need to be considered. So far, most of the numerical simulation models developed for three-dimensional wave propagation have been built upon the potential flow theory. Using integral equation methods, highly accurate numerical models have been developed for wave propagation over varying bathymetry in shallow water and for wave-body interaction in deep water $[3,4,5]$. However, the potential flow assumption limits these models' applications to irrotational flow.

Relaxing the potential flow assumption, the three-dimensional Navier-Stokes (N-S) equations can be employed to describe flow motions. Theoretically, the direct numerical simulation (DNS) can always be performed to resolve the entire spectrum of motions ranging from large eddy motions to the smallest turbulence (Kolmogorov) scale motions. Clearly, the DNS requires very fine spatial and temporal resolutions and most of DNS applications can only be applied to relatively low Reynolds number flows within a small computational domain [6]. With the currently available computing resources, the DNS is still not a feasible approach for investigating wave-body interaction problems if wave breaking and flow separation are important.

The alternatives to the DNS approach for computing the turbulent flow characteristics include the Reynolds Averaged Navier-Stokes (RANS) equations method and the Large Eddy Simulation (LES) method. In the RANS equations method, only the ensemble-averaged (mean) flow motion is resolved. The turbulence effects appear in the momentum equations for the mean flow and are represented by the Reynolds stresses, which are often modeled by an eddy viscosity model. The eddy viscosity can be further modeled in several different closures [7]. For example in the $k-\varepsilon$ closure model, the eddy viscosity is hypothesized as a function of the turbulence kinetic energy $(k)$ and the turbulence dissipation rate $(\varepsilon)$, for which balance equations are constructed semi-empirically. Lin and Liu [8] have successfully applied the $k-\varepsilon$ turbulence model in their studies of wave breaking and runup in the surf zone. Lin and Liu's model has been extended and applied to many different coastal engineering problems, including the wavestructure interaction [9]. In the LES method, the three-dimensional turbulent motions are directly simulated and resolved down to a pre-determined scale, and the effects of smaller-scale motions are then modeled by closures. In terms of the computational expense, LES lies between RANS and DNS. Compared to DNS in solving high-Reynolds-number flows, LES avoids explicitly representing small-scale motions and therefore, the computational costs can be greatly reduced. Compared to RANS models, because the large-scale unsteady motions are computed explicitly, LES can be expected to provide more statistical information for the turbulence flows in which large-scale unsteadiness is significant [7].

The flow governing equations for LES are derived from the N-S equations by applying a low-pass spatial filter. Similar to the RANS approach, a term related to the residual-stress tensor or the sub-grid-scale (SGS) Reynolds stress tensor appears in the filtered N-S equations. Thus, a closure model is also required to relate the residual-stress tensor to the filtered velocity field. The traditional Smagorinsky model [10] is probably the simplest LES-SGS model and has been used in several breaking wave studies $[11,12,13,14]$.

In the present study, we plan to extend the LES model developed by Liu et al. [14], which was originally designed for studying landslide generated tsunamis, to wave-pile interaction problems. The model solves the filtered NS equations using a two-step projection algorithm with finite volume formulation. The Volume-of-Fluid (VOF) method [15] is used to track the free surface motions. The Smagorinsky SGS model is employed in [14]. In the present work, we 
intend to improve the SGS model by implementing a dynamic SGS closure scheme. However, before developing an adequate dynamic SGS model, we would like to verify first that the core algorithms of the model (i.e., the VOF method and the two-step projection methods) are adequate for dealing with the wave-piles interaction problems. Thus, in this paper, we will ignore the viscous and turbulent effects and solve only the Euler's equations. The algorithms for inputting the appropriate incident waves and for the outgoing waves through a boundary are developed.

Therefore, the objectives of this paper are: (i) the validation of a three-dimensional numerical model based on the Euler's equations for non-breaking wave propagation and (ii) the investigation of the influence of a pile on the wave field. For this purpose laboratory data sets, containing large-scale measurements of the water surface elevation, the fluid particle velocity, the pressure at different locations around the circumference of the pile, and the total wave force are used to check the accuracy of the numerical results

\section{The Numerical Model}

Fluid motion of incompressible and inviscid fluid can be described by the Euler's equations:

$$
\begin{gathered}
\nabla \cdot \mathbf{u}=0 \\
\frac{\partial \mathbf{u}}{\partial t}+\nabla \cdot(\mathbf{u u})=-\frac{1}{\rho} \nabla p+\mathbf{g}
\end{gathered}
$$

where $\mathbf{u}$ represents velocity vector, $\rho$ water density, $\mathbf{g}$ the gravity force vector, $t$ time, and $p$ pressure.

To simulate the wave-pile interaction in a wave flume, the computational domain is usually a rectangular box which is occupied by water, air and the pile. The upper (ceiling) and lower (bottom of the flume) boundaries and two lateral (sidewalls of the flume) boundaries of the boundaries as well as the surface of the pile are rigid boundaries. Therefore, the no-flux boundary condition is applied. The incident wave information, including the velocity and the free surface displacement, are input at the upstream boundary, while an outflow boundary condition is applied at the downstream boundary. On the free surface, the dynamic boundary condition requires that the pressure filed is continuous. On the other hand, the kinematic boundary condition is replaced by requiring the conservation of a volume of fluid function, $f$, representing the volume fraction of water within a computational cell. The $f$ value equals to one, if the cell is full, zero if empty, and $0<f<1$ if the cell is partially filled with water. The governing equation for $f$ can be described by:

$$
\frac{\partial f}{\partial t}+\nabla \cdot(f \mathbf{u})=0
$$

The Euler's equations are solved by the two-step projection method [16]. The momentum equations, (2.2), are split into two fractional steps:

$$
\frac{\rho^{n+1} \mathbf{u}^{*}-\rho^{n} \mathbf{u}^{n}}{\Delta t}=-\nabla \cdot\left(\rho^{n} \mathbf{u u}\right)^{n},
$$




$$
\frac{\rho^{n+1} \mathbf{u}^{n+1}-\rho^{n+1} \mathbf{u}^{*}}{\Delta t}=-\nabla p^{n+1}+\rho^{n+1} \mathbf{g},
$$

in which the superscript " $n$ " denotes the $n$-th time step. Equation (2.4) is an explicit expression for the interim velocity, $\mathbf{u}^{*}$, referred to as the predictor step. On the other hand, (2.5) is called the projection step. Combining (2.4) with (2.5) produces the time discretization of (2.2):

$$
\frac{\rho^{n+1} \mathbf{u}^{n+1}-\rho^{n} \mathbf{u}^{n}}{\Delta t}=-\nabla \cdot(\rho \mathbf{u u})^{n}-\nabla p^{n+1}+\rho^{n+1} \mathbf{g} .
$$

No additional approximation results from this decomposition.

Equation (2.5) relates $\mathbf{u}^{n+1}$ to $\mathbf{u}^{*}$. By adopting the continuity condition, (2.1), we have:

$$
\nabla \cdot \frac{\nabla p^{n+1}}{\rho^{n+1}}=\nabla \cdot\left(\frac{\mathbf{u}^{*}}{\Delta t}+\mathbf{g}\right)
$$

The above equation is also called the Poisson Pressure Equation (PPE). The pressure $p^{n+1}$ at the new time step can be obtained by solving (2.7).

The two-step projection method is implemented in a finite volume algorithm so that unstructured computational grids (cells) can be used. We also note that a multidimensional PLIC (piecewise linear interface calculation) method [17] is utilized to construct the free surface. The details of the algorithm can be found in $\mathrm{Wu}[16]$.

\section{Comparison of Laboratory Data and Numerical Results}

To check the capability and accuracy of our current numerical model, we compare numerical results with available experimental data. The numerical simulations of non-breaking periodic waves and solitary waves and their interaction with a cylindrical pile were conducted and the results were compared with the experiments conducted in the Large Wave Flume (GKW) of the Coastal Research Centre (FZK) in Hannover, Germany.

\subsection{Laboratory Set-Up in the GKW Experiments}

A series of large-scale experiments were conducted in the Large Wave Flume (GKW) of the Coastal Research Centre (FZK) in Hannover, Germany, to measure the wave forces acting on a cylindrical pile. This wave flume has an effective length of $309 \mathrm{~m}$, a width of $5 \mathrm{~m}$ and a depth of $7 \mathrm{~m}$. A steel circular cylinder with a diameter of $0.7 \mathrm{~m}$ was instrumented and installed in the flume. This paper deals only with a series of tests of non-breaking monochromatic and solitary waves with the pile placed on flat bottom of the flume at a distance of $111 \mathrm{~m}$ from the wavemaker (see Figure 2). Additional details on the test set-up are given in [18] and [19]. 


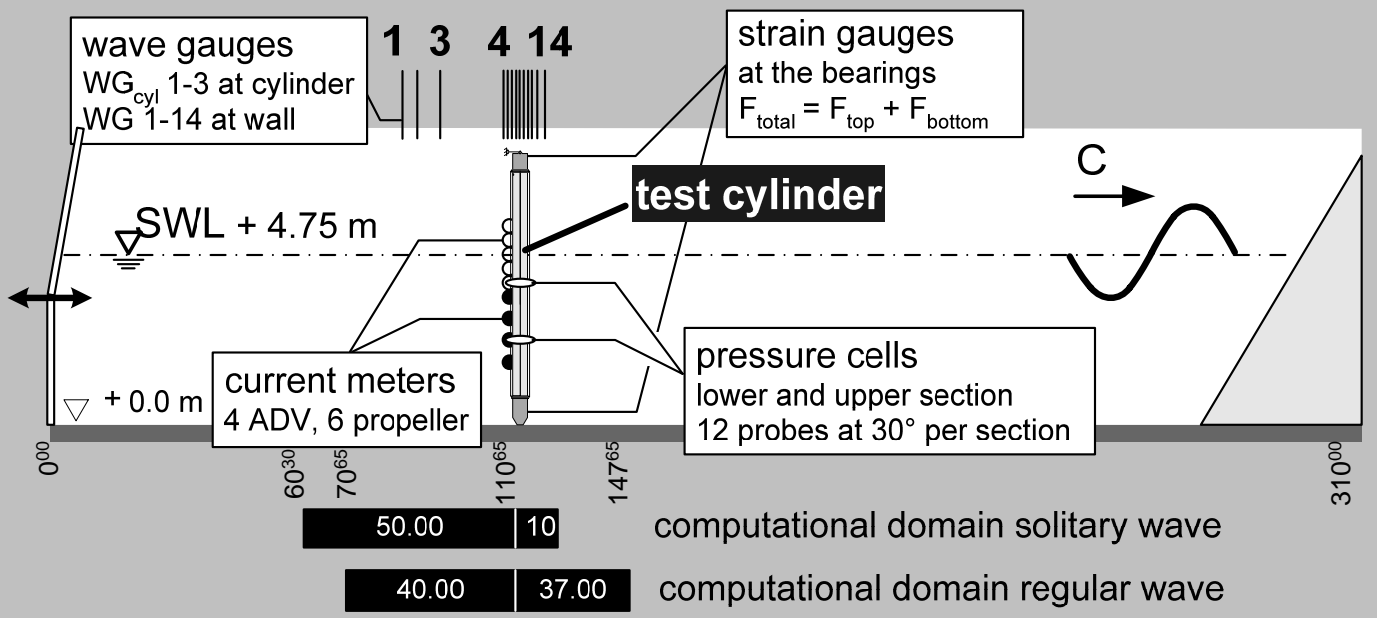

Figure 2 A sketch of the Large Wave Flume (GKW) of the Coastal Research Centre (FZK) with a summary the measuring devices used for comparison in this study

To measure wave forces, strain gauges were installed at the two bearings (top and bottom) of the cylinder. Additionally, 49 pressure transducers were fixed on the cylinder; they are uniformly distributed along the frontline of the cylinder with a spacing $\Delta z=0.2 \mathrm{~m}$ and are also spread out over the circumference in two horizontal cross sections. The time histories of the water surface elevation were measured with 19 wave gauges. Four wave gauges were located at the pile and 15 of them along the flume. Four two-component current meters measured the water particle velocities under the trough and six propeller probes are used in the region above the wave trough. They were located at the side wall of the flume corresponding to the front line of the cylinder.

In this paper three representative experimental cases are used to check the numerical results in detail. The characteristics of these non-breaking regular waves and solitary wave cases, ranging from intermediate water depth waves to the shallow water wave, are shown in Table 1.

\begin{tabular}{|c|c|c|c|c|c|c|}
\hline & $\begin{array}{c}\text { type of } \\
\text { wave }\end{array}$ & $\begin{array}{c}\text { water } \\
\text { depth, } \\
\boldsymbol{h}[\mathrm{m}]\end{array}$ & $\begin{array}{c}\text { wave } \\
\text { period, } \\
\boldsymbol{T}[\mathbf{s}]\end{array}$ & $\begin{array}{c}\text { Wave } \\
\text { height, } \\
\boldsymbol{H}[\mathbf{m}]\end{array}$ & $\boldsymbol{k h}$ & $\boldsymbol{k H}$ \\
\hline $\mathbf{1}$ & regular & $\mathbf{4 . 7 6}$ & $\mathbf{4}$ & $\mathbf{1 . 2 0}$ & $\mathbf{1 . 3 5 2 3}$ & $\mathbf{0 . 3 2 8 2}$ \\
\hline 2 & regular & 4.77 & 6 & 1.35 & 0.7885 & 0.2233 \\
\hline 3 & solitary & 4.755 & 4.92 & 0.7 & - & - \\
\hline
\end{tabular}

Table 1. Wave conditions used in the laboratory experiments (GWK) and numerical simulations

\subsection{Numerical simulations of GWK solitary wave experiments}

To reduce the computational costs, it is desirable to use only half width of the wave flume in the numerical simulations. The concern, however, is whether the flow field is actually symmetric with respect to the centreline of the tank. We have compared the results based on the full wave tank width as well as the half wave tank width. The differences between two simulations are negligible. Therefore, all the results presented herein are based on the half wave tank width simulations. As shown in Figure 3, the horizontal computational domain is $60 \mathrm{~m}$ long and $2.5 \mathrm{~m}$ wide and is bounded laterally by two solid boundaries. The incident wave conditions are prescribed along the upstream boundary, while the radiation boundary condition is employed at 
the downstream boundary. The wave is allowed to propagate out of the computational domain without reflection by specifying the outgoing flow properties:

$$
\frac{\partial \varphi}{\partial t}+C_{g} \frac{\partial \varphi}{\partial n}=0
$$

where $\varphi$ is a physical variable, such as the fluid velocity, $C_{g}$ is the group velocity and $C_{g}=\sqrt{g(h+\eta)}$ for a long wave, $\eta$ is the free surface elevation measured from the still water depth, and $h$ the still water depth; $\partial \varphi / \partial n$ is the normal derivative at the downstream boundary.

Unstructured meshes are used to discretize the computational domain with finer grids in the region near the cylinder. Generally speaking, 10 grids with uniform grid size, $\Delta y=0.25 \mathrm{~m}$, are placed in the spanwise direction and 59 non-uniform grids with minimum grid $\Delta z_{\min }=0.05 \mathrm{~m}$ near the free surface are installed in the vertical direction. In the streamwise direction three different zones are designed: Near the upstream boundary coarser grids with $\Delta x=0.6 m \sim 0.25 m$ are installed; in the neighbourhood of the pile finer grids, $\Delta x=0.25 m \sim 0.06 m$, are employed (There are 18 grids along the half perimeter of the pile); and coarser grids, $\Delta x=0.06 m \sim 0.5 m$ are employed near the outflow boundary. The total number of computational cells is 101,716.

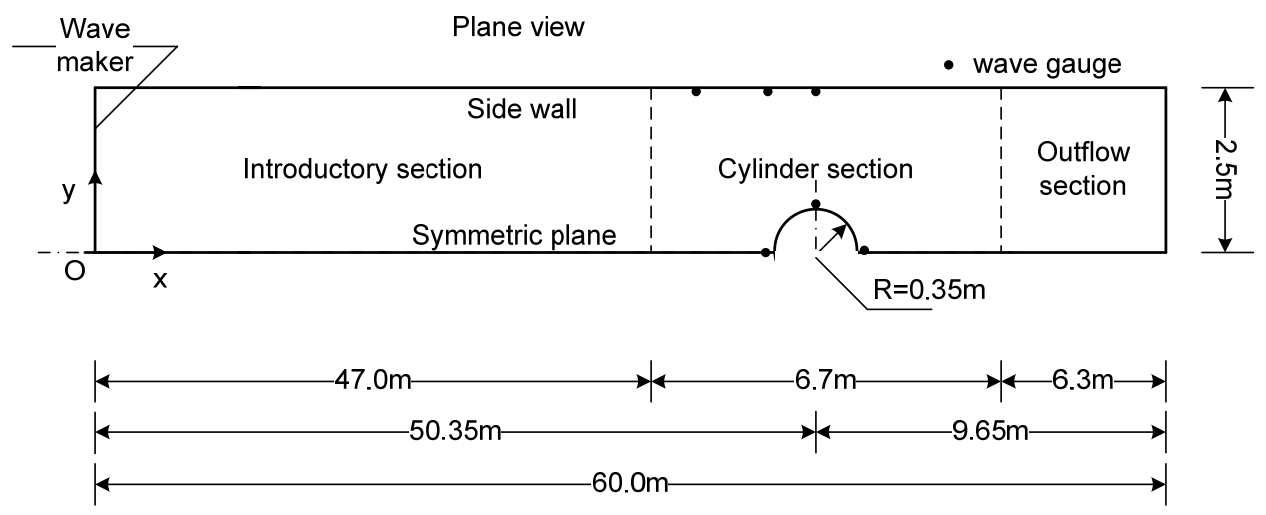

Figure 3 Computational domain (not to scale)

The experimentally measured time history of free surface displacements at the wave gauge located at $29.5 \mathrm{~m}$ upstream of the pile was used as the input incident wave boundary condition at the upstream boundary. The corresponding fluid velocity for the incident wave is calculated using the following approximate formula [20]:

$$
\begin{aligned}
& \frac{u}{\sqrt{g h}}=\frac{\eta}{h}\left[\frac{H}{h}+3\left(\frac{H}{h}\right)^{2}\left(\frac{1}{6}-\frac{1}{2}\left(\frac{z}{h}+1\right)^{2}\right)\right]-\left(\frac{H}{h}\right)^{2}\left(\frac{7}{4}-\frac{9}{4}\left(\frac{z}{h}+1\right)^{2}\right)\left(\frac{\eta}{h}\right)^{2}, \\
& \frac{w}{\sqrt{g h}}=\sqrt{\frac{3 H}{h}}\left(\frac{z}{h}+1\right) \frac{\eta}{h} \tanh \left(\sqrt{\frac{3 H}{4 h^{3}}}(x-C t)\right)\left\{1+\frac{H}{2 h}\left[1-7 \frac{\eta}{H}-\left(\frac{z}{h}+1\right)^{2}\left(1-\frac{3 \eta}{H}\right)\right]\right\},
\end{aligned}
$$

where $\eta$ denotes the measured time history of free surface elevation, $H$ the wave height and $h$ the water depth. 
Figure 4 shows the numerical results for the time histories of free surface displacement at several wave gauge locations. The time used in these figures indicates the numerical simulation time. An excellent agreement between the numerical results and the experimental data for the wave crest and the trailing wave trough is observed at gauges in front of the pile. However, noticeable differences near the wave crest at the wave gauge, located at $10 \mathrm{~cm}$ away from the side of the pile ( $\theta=90^{\circ}$ ), are observed, which could be the consequence of flow separation.



Figure 4 Time histories of free surface displacements.

Figure 5 shows the time histories of the horizontal particle velocity at different locations. ADV velocity meters are installed at the side wall corresponding to the frontline of the pile. ADV $1 \sim 4$ and the propeller probes are located at different elevations and the elevations from the bottom are $z_{\mathrm{ADV} 1}=2.03 \mathrm{~m}, z_{\mathrm{ADV} 3}=3.23 \mathrm{~m}, z_{\mathrm{ADV} 4}=3.83 \mathrm{~m}, z_{\text {prop } 1}=4.23 \mathrm{~m}, z_{\text {prop } 2}=4.63 \mathrm{~m}$, $z_{\text {prop3 }}=5.03 \mathrm{~m}$, respectively. We first note that the experimental data are raw data without applying any filtering out the noise. The numerical results for the maximum horizontal velocity slightly over-estimate the laboratory data. It is interesting to observe that although trailing wave troughs appeared in the free surface measurements shown in Figure 4 these troughs disappeared in the horizontal velocity measurements. The numerical results are consistent with the surface elevation as shown in Figure 4. Additionally, the agreement between the experimental data and numerical results is incredibly good for the vertical component (which is not shown here). 


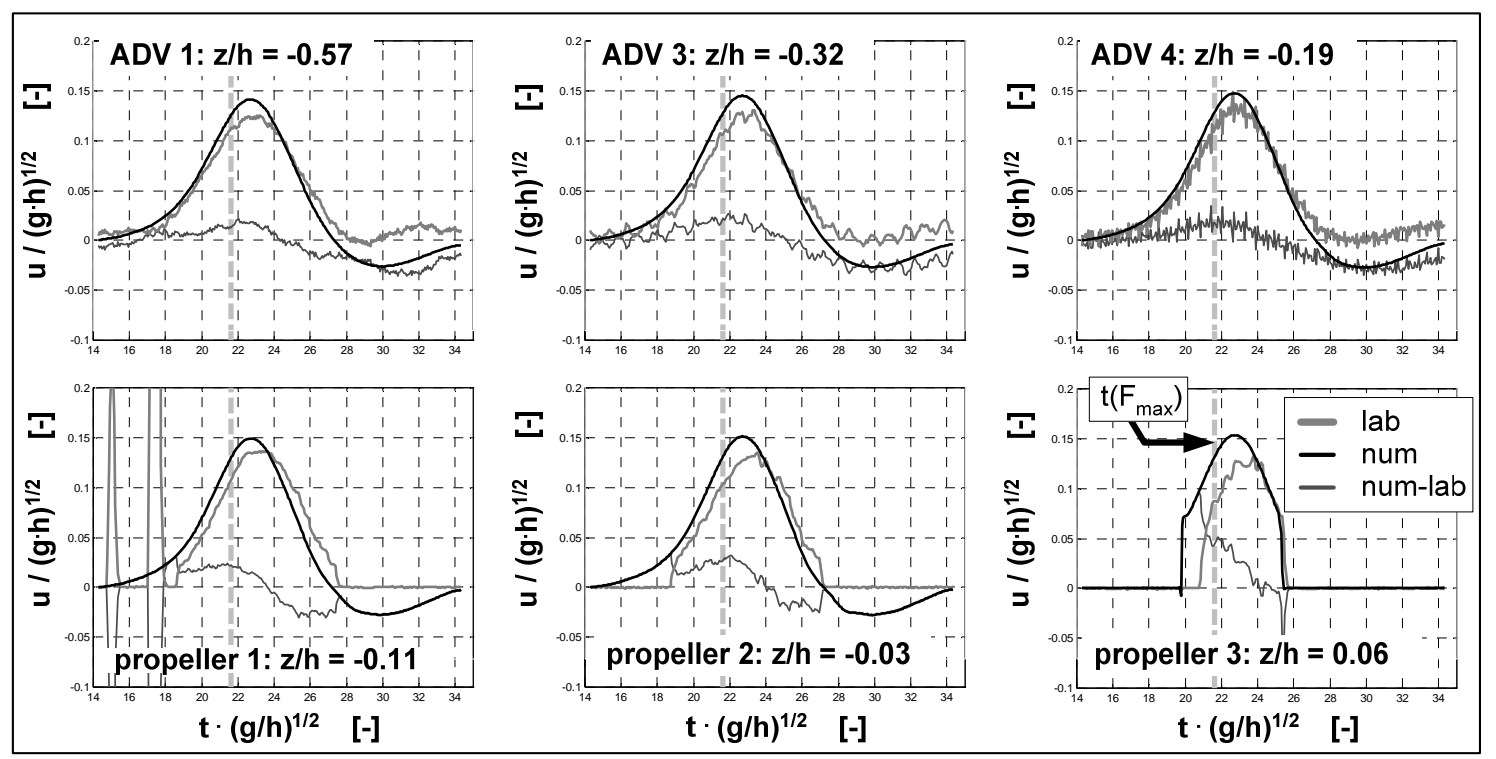

Figure 5 Time histories of horizontal particle velocity at different elevations.

In Figure 6 the dynamic pressure responses along the perimeter at $z / h=-0.11(4.23 \mathrm{~m}$ above the bottom) are plotted. Note that $\theta=0^{\circ}$ and $180^{\circ}$ denote the front and back of the pile, respectively. The agreement between the numerical results and experimental data is excellent except at the locations $\theta=0^{\circ}$ and $120^{\circ}$. Similar agreement is also obtained along the perimeter at $\mathrm{z} / \mathrm{h}=-0.44$ (2.63 $\mathrm{m}$ above the bottom), which is not shown here. Figure 7 shows the dynamic pressure distribution over the depth in the front of the pile, $\theta=0^{\circ}$, at the instant $t \sqrt{g / h}=21.62$ when the force acted on the pile reaches its maximum. The dynamic pressure varies nearly linearly above the still water level and is almost a constant under the still water level. The numerical results overestimate the experimental data by about $10 \%$ under the still water level, while the agreement is very good above the still water level.

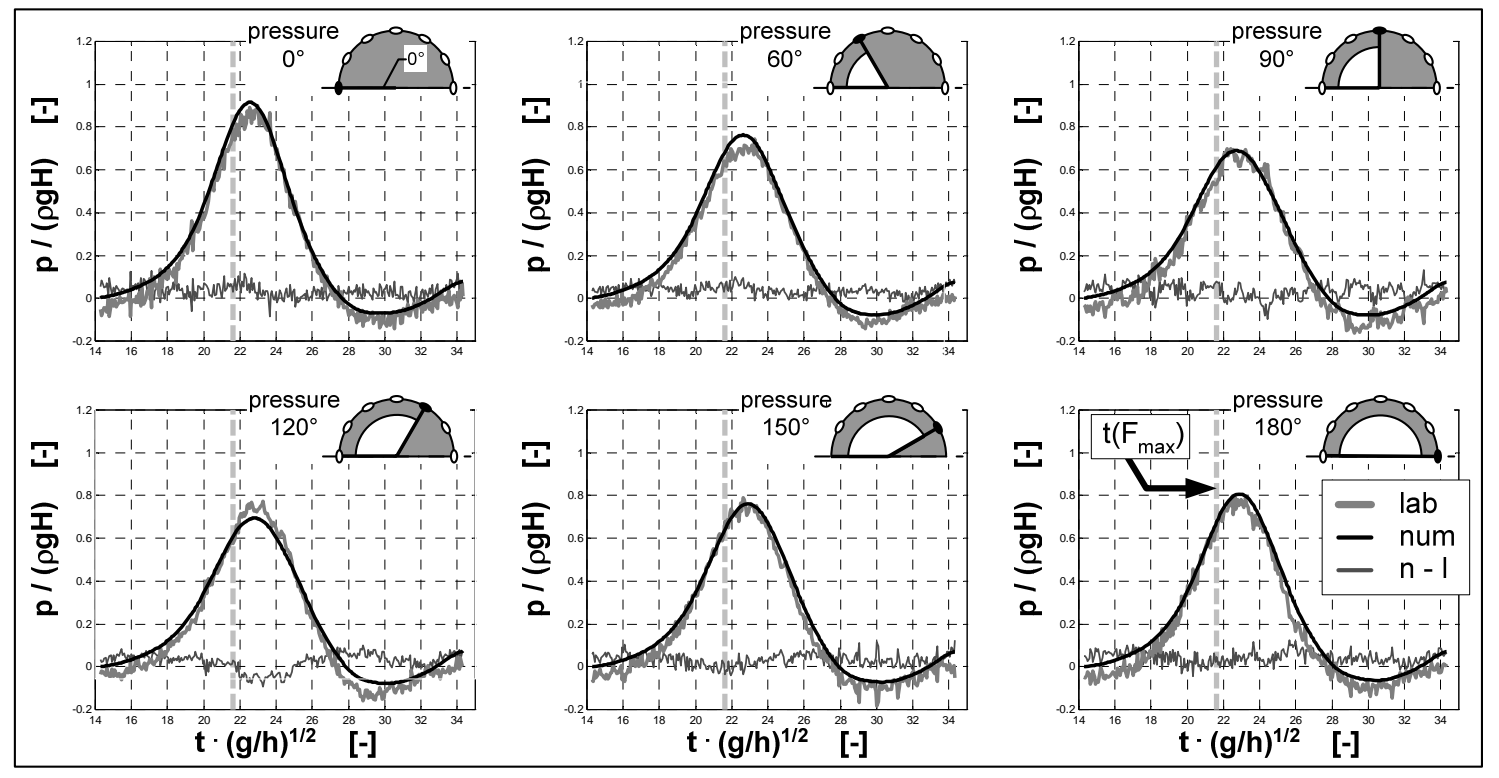

Figure 6 Time histories of dynamic pressure along the perimeter of the pile at $\mathrm{z} / \mathrm{h}=\mathbf{- 0 . 1 1}$. 


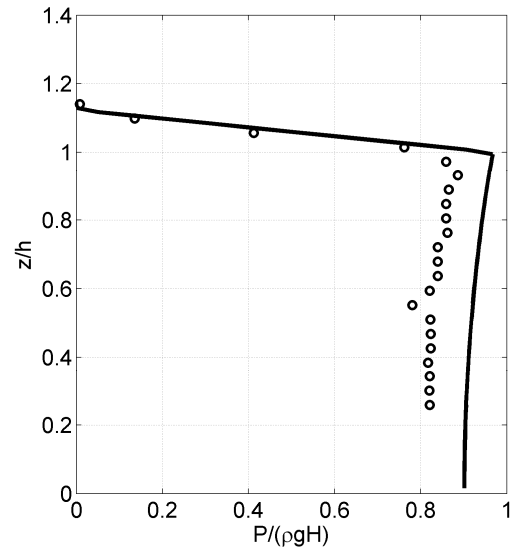

Figure 7 Dynamic pressure over the depth in front of the pile (Solid line: numerical; $\bigcirc$ : laboratory).

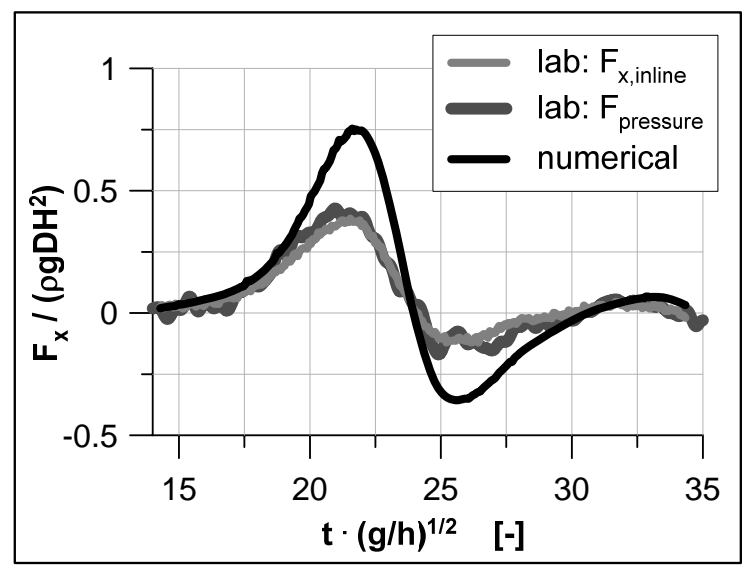

Figure 8 Time history of total wave force acting on the pile.

Figure 8 shows the measured and calculated wave force acting on the cylinder. Numerical results are obtained by integrating the pressure distribution on the wetted surface of the pile. The agreement is surprisingly poor; the difference in maximum force is almost a factor of 2. In view of the good agreement between experimental data and numerical results for velocity, pressure and free surface elevation as shown in Figures 4, 5, and 6, the only explanation for such a huge difference in resulting force could be a mistake made in our numerical integration of pressure. However, as to be shown in the next section, the same algorithm is used to calculate the wave forces due to periodic waves. A much better agreement is observed. Therefore, the question for the discrepancy shown in Figure 8 remains open.

\subsection{Numerical simulations of GWK periodic wave experiments}

Numerical simulations were also carried out for two non-breaking periodic wave cases as shown in Table 1. The detailed comparisons are presented here only for the first case with a water depth $h=4.76 \mathrm{~m}$, a wave period $T=4.0 \mathrm{~s}$ and a wave height $H=1.2 \mathrm{~m}$. In this section first the incident boundary conditions are discussed and the technique is checked due to the comparison of numerical results with the so called undisturbed measured water surface elevations and horizontal particle velocities in the next section. Finally, the loads on the slender pile, i.e. local pressure and total force, from numerical and laboratory experiments will be shown.

In order to minimize the computational domain and to use the available computer source most efficiently, a regular wave train is driven into the numerical tank directly on the incident boundary (see Figure 3) by specifying appropriate water surface elevation, horizontal and vertical particle velocity in the entire water depth. These quantities are obtained by adopting the Fourier approximation method suggested by Fenton [21] with the wave parameters indicated in Table 1. Thus, the wave free surface elevation and fluid particle velocities at incident wave boundary $(x=0)$ are:

$$
\begin{aligned}
& \eta(t)=\frac{1}{k}\left[\sum_{j=1}^{N} Y_{j} \cos j k C t\right] \\
& u=\frac{1}{\sqrt{k / g}}\left[C \sqrt{k / g}-\bar{U} \sqrt{k / g}+\sum_{j=1}^{N} j B_{j} \frac{\cosh j k(h+z)}{\cosh j k h} \cos j k C t\right]
\end{aligned}
$$




$$
w=\frac{1}{\sqrt{k / g}}\left[\sum_{j=1}^{N} j B_{j} \frac{\sinh j k(h+z)}{\cosh j k h} \sin j k C t\right]
$$

The spanwise velocity component, $v$, is zero at the incident wave boundary condition. The values of Fourier coefficients $Y_{j}$ and $B_{j}$ depend on the choice of truncation order. The parameter $C$ is the wave celerity and $\bar{U}$ is the mean fluid speed in the moving frame with a speed of $C$. The advantage of all Fourier approximation methods, e.g. [21] and [22], is that these methods can be applied to general wave conditions, including waves in both deep water and water of finite depth. The Fenton formulation can adopt both Stokes first and second approximation of phase speed. For the present simulation in a closed wave tank Fenton's method uses the Stokes second approximation of phase speed together with zero Stokes drift $C_{S}$.

The computational domain for this simulation is slightly larger than the one used for the solitary wave simulations. The total length in the direction of wave propagation is $77 \mathrm{~m}$, where the distance between the upstream boundary and the front of the cylinder is $40 \mathrm{~m}$ long. Finer grids are installed around the cylinder with 26 grid points along the perimeter of the pile and coarser grids are used in the regions far away from the pile with $\Delta x=0.35 \mathrm{~m}$ (roughly, 60 grids in one wavelength). The vertical grid is uniform with $\Delta z=0.1094 \mathrm{~m}$, i.e., there are about 10 grids within one wave height.

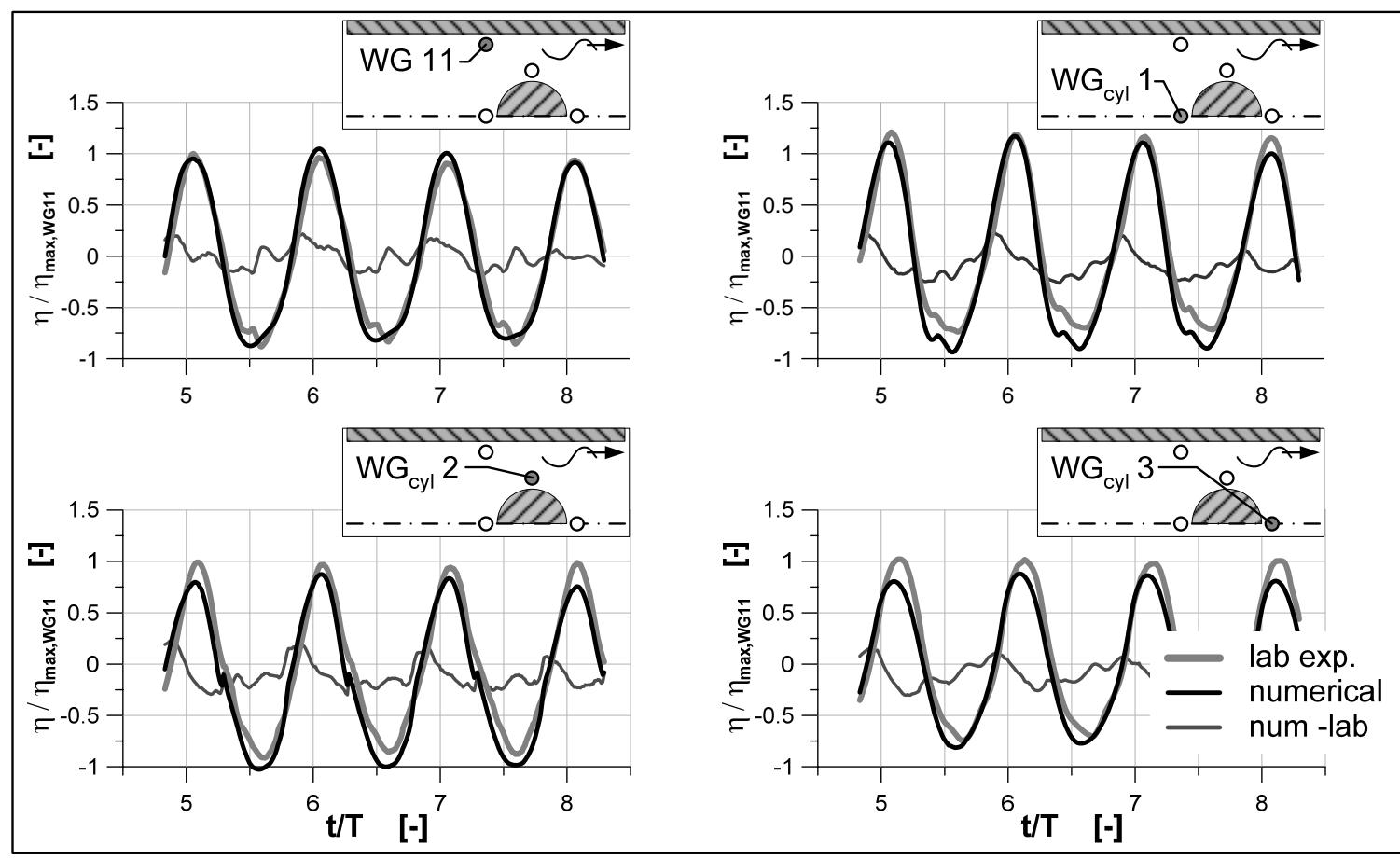

Figure 9 Free surface displacements at various locations upstream and in the vicinity of the pile.

The surface elevations measured at several wave gauges are plotted with numerical results in Figure 9. Incident wave condition measured at the flume wall (WG 11) and the water surface elevation at the cylinder $\left(\mathrm{WG}_{\mathrm{CYL}}-1 \sim 3\right)$ are shown. The synchronization of all time histories has been done due to the comparison of numerical and laboratory surface elevation measured at wave gauge WG 11. The reference wave amplitude in Figure 9 is the maximum


results is reasonably good. Even some characteristic ripples are predicted well, which are measured in the frontline of the cylinder $\left(\mathrm{WG}_{\mathrm{cyl}} 1\right)$ while the wave trough passes the pile. 


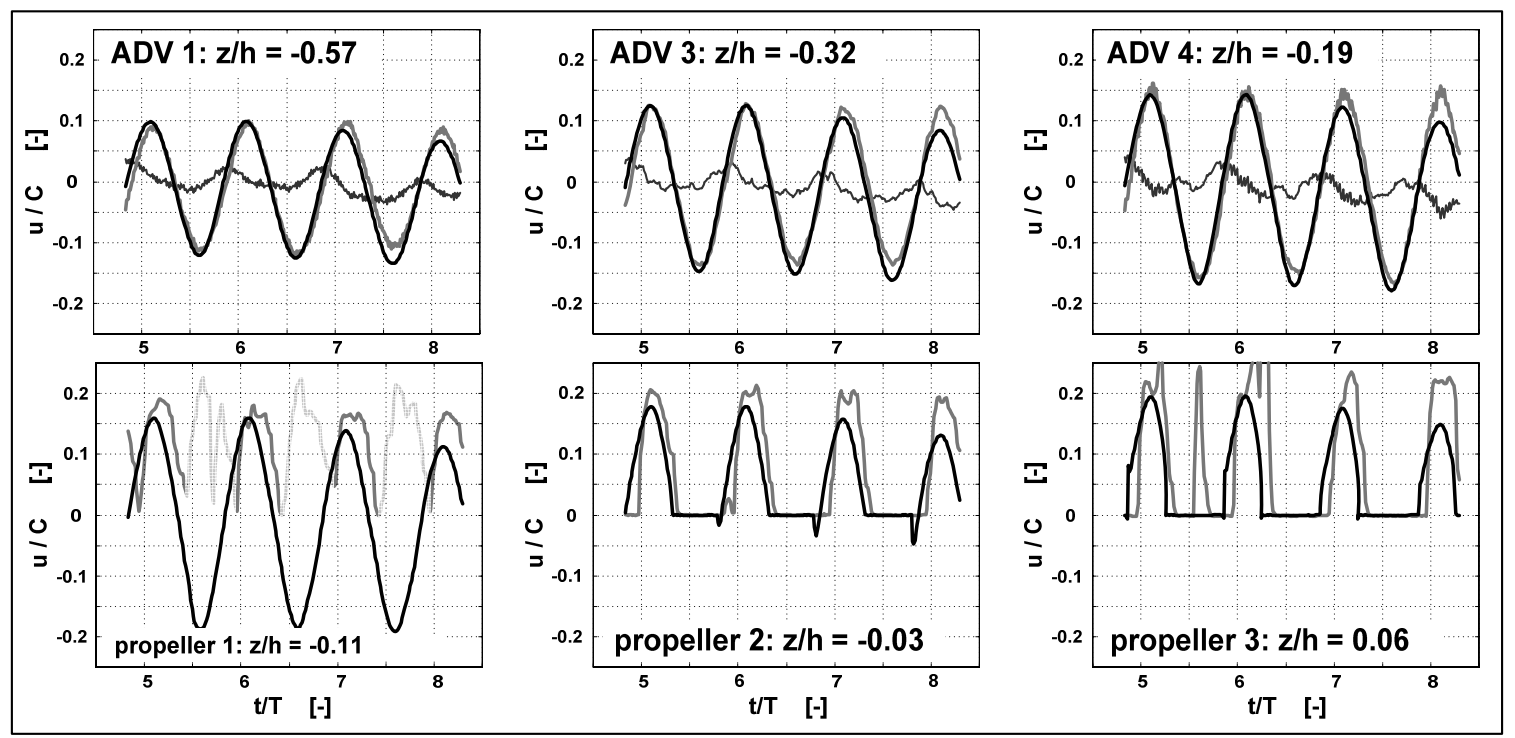

Figure 10 Time histories of horizontal particle velocity at different elevations.

Figure 10 shows the velocity profiles at different elevations from the bed. The numerical model tends to underestimates the crest of the velocity slightly, while overestimates the trough velocities. It is obvious that the propeller probes record absolute values only. The numerical results show a downward drift, suggesting a small opposing current. This feature could be caused by the imperfect incident boundary conditions.
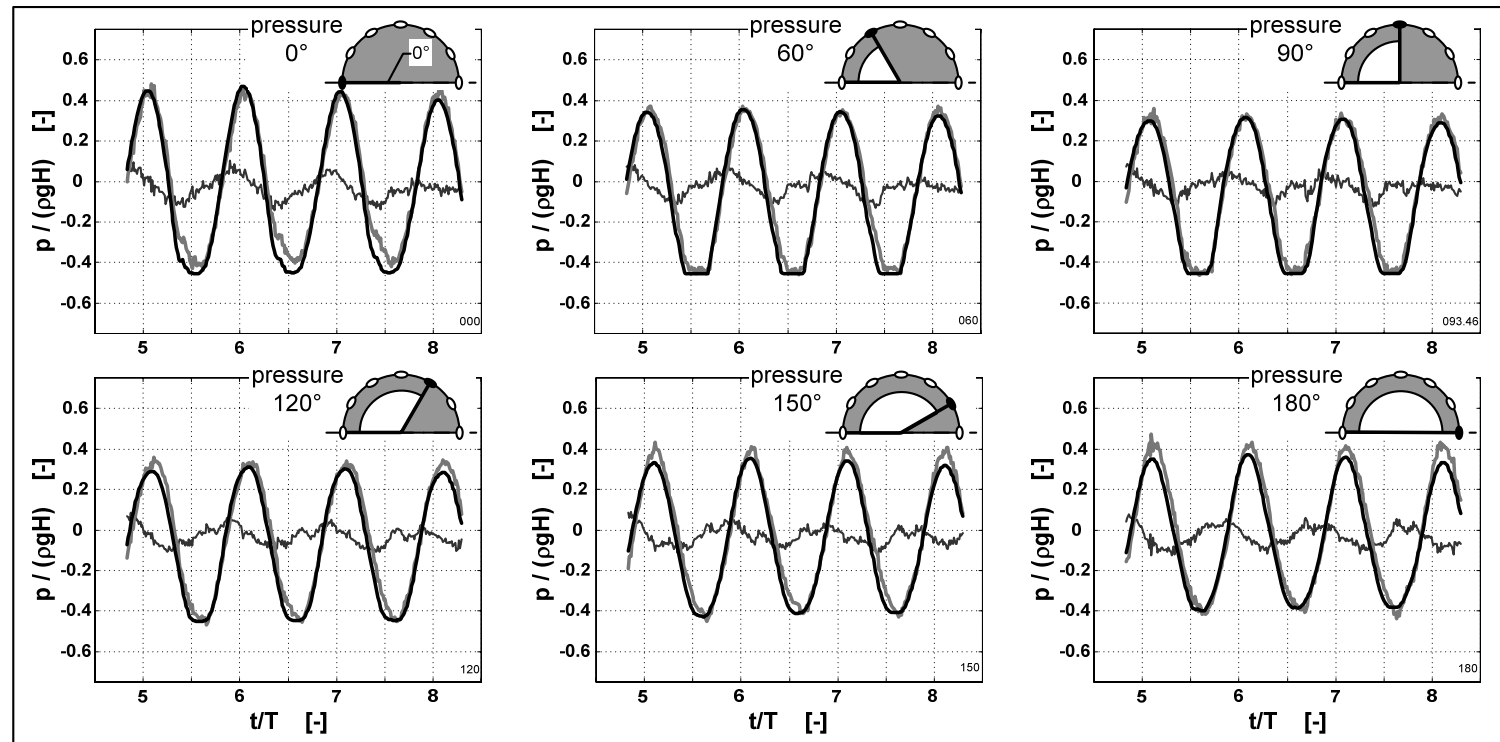

Figure 11 Time histories of dynamic pressures along the perimeter of the pile at $\mathrm{z} / \mathrm{h}=\mathbf{- 0 . 1 1}$.

The time histories of dynamic pressure along the perimeter of the pile at $\mathrm{z} / \mathrm{h}=-0.11$ (4.23 $\mathrm{m}$ above the bottom) are shown in Figure 11. The numerical model underestimates most of the crests of the measured pressure slightly. Taking into account the comparison of the incident wave conditions, the difference of the measured and predicted pressure is in agreement with the differences presented in Figure 10. The critical region from view of flow separation, $\theta=90^{\circ}-120^{\circ}$, no significant change of the time history is visible, neither for the comparison 
between each laboratory experiment nor for the comparison with the numerical results. The pressure measurements of the lower section have the same pattern as shown here for the upper section.

The time history of the total inline force acting on the pile is plotted on the left hand side in Figure 12. In the case of the laboratory data the force $F_{x}$ represents the sum of the measured force in the bearings and in the numerical case the pressure has been integrated over the wetted surface of the cylinder. The total inline force is predicted with excellent accuracy. The maximum discrepancy in the maximum force is about $10 \%$. Recall that in the solitary wave case the difference in wave force is a factor of two, although the overall agreement between the numerical results and experimental data for the velocity, free surface elevation and pressure is much better than that for the periodic wave case.

To proof the assumption that the determined test is inertia dominated, a comparison with the Morison-Inertia force using a force coefficient $C_{M}=2$ and the undisturbed water surface elevation (measured at the side wall) is shown on the right hand side of Figure 12. The extreme loads occur synchronously to the zero-crossings of the water surface elevation and are in phase with the horizontal particle acceleration.

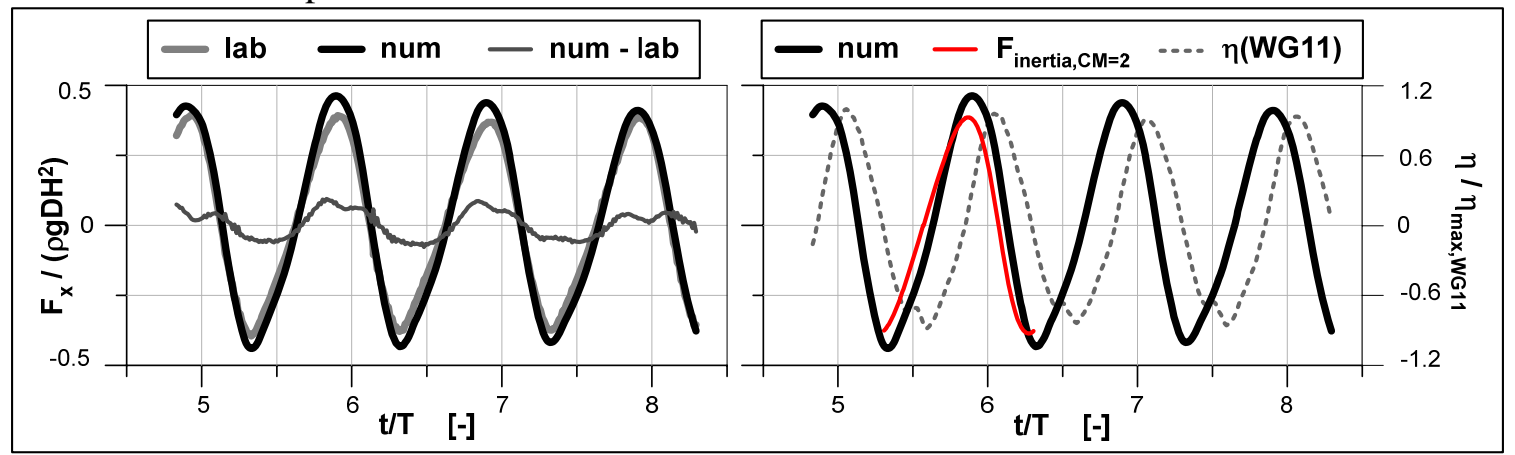

Figure 12 Time history of the total force acting on the pile.

\section{Concluding Remarks}

A 3D numerical model, developed for studying water wave-body interaction problems, has been tested with experimental data for periodic and solitary waves interacting with a slender pile. For the tested cases, waves are non-breaking and turbulence is negligible. The comparisons show that the numerical model is capable of predicting free surface displacement, fluid particle velocity, and dynamic pressure on the pile, provided that the correct incident boundary conditions are applied. The relatively less satisfactory agreement in the particle velocity of periodic waves may be due to the imperfect incident boundary conditions used in the numerical simulations. A ramp function, i.e. spinning up the incident waves, can be used to improve the situation.

The agreement between experimental data and numerical results for the wave force is reasonable and consistent with the comparisons of other physical variables for the periodic cases. Unfortunately, this is not the case for solitary waves. Large discrepancy is observed in the wave force measurements and numerical calculations. Additional numerical and laboratory experiments will be performed in the near future.

\section{Acknowledgement}

We would like to acknowledge the support from National Science Foundation of the United States through various research grants to Cornell University. The support received from DFG for the basic research project "breaking wave loads on slender cylindrical piles" (OU 1/4-1 
\&-2) is also gratefully acknowledged by the authors. Kai Irschik would like to acknowledge the support received from the German Academic Exchange Service, which enabled him to visit Cornell University.

\section{REFERENCES}

[1] Morison, J. R., O’Brien, M.P., Johson, J. W., and Schaaf, S. A. 1950 The forces exertd by surface waves on piles. J. Petroleum Technology, Petroleum Transctions, AIME, 189, 149154.

[2] Sapkaya, T. and Issacson, M. St. Q. 1981 Mechanics of Wave Forces on Offshore Structures, Van Nostrand Reinold, New York.

[3] Xue, M., Xu, H. Liu, Y. and Yue, D. K. P. 2001 Computations of fully nonlinear three dimensional wave-wave and wave-body interaction. Part 1. Dynamics of steep threedimensional waves. J. Fluid Mech., 438, 11-39.

[4] Liu, Y., Xue, M. and Yue, D.K.P. 2001 Computations of fully nonlinear three-dimensional wave--wave and wave--body interactions. Part 2. Nonlinear waves and forces on a body, $J$. Fluid Mech., 438 , 41 - 65.

[5] Guyenne, P. and Grilli, S. T. 2006 Numerical study of three-dimensional overturning waves in shallow water. J. Fluid Mech.,

[6] Kim, J., Moin P., and Moser R. 1987 Turbulence statistics in fully developed channel flow at low Reynolds number. J. Fluid Mech., 177, 133-166.

[7] Pope, S. B. 2001 Turbulent flows. Cambridge University Press.

[8] Lin, P. and Liu, P. L.-F. 1998a A numerical study of breaking waves in the surf zone. J. Fluid Mech., 359, 239-264.

[9] Liu, P. L.-F., Lin P. Z. and Chang, K. A. 1999 Numerical modeling of wave interaction with porous structures, J. Wtrwy., Port, Coast., and Oc. Engng., ASCE 125 (6): 322-330.

[10] Smagorinsky, J. 1963 General circulation experiments with the primitive equations: I. The basic equations. Mon. Weather Rev. 91, 99-164.

[11] Watanabe, Y. and Saeki, H. 1999 Three-dimensional large eddy simulation of breaking waves, Coasal. Engng., 41 (3/4), 281-301.

[12] Lin, P. and Li, C. W. 2002 A $\sigma$-coordinate three-dimensional numerical model for surface wave propagation. Int. J. Numer. Meth. Fluids, 38:1048-1068.

[13] Christensen, E. D., and Deigaard R. 2001 Large eddy simulation of breaking waves, Coastal Engng. 42, 53-86.

[14] Liu, P. L.-F., Wu, T.-R., Raichlen, F., Synolakis, C. E., and Borrero, J. C. 2005 Runup and rundown generated by three-dimensional sliding masses, J. Fluid Mech., 536: 107-144.

[15] Hirt, C. W. and Nichols, B. D. 1981 Volume of fluid (VOF) method for the dynamics of free boundaries. J. Comp. Phys., 39, 201-225.

[16] WU, T.-R., 2004 A numerical study of three-dimensional breaking waves and turbulence effects. PhD dissertation, Cornell University.

[17] Rider, W. J. and Kothe, D. B. 1998 Reconstructing Volume Tracking, J. Comp. Phys., 141, 112-152.

[18] Wienke, J. and Oumeraci, H. 2005 Breaking wave impact force on a vertical and inclined slender pile. Coastal Engineering. 52, 435-462.

[19] Irschik, K., Sparboom, U. and Oumeraci, H. 2003 Breaking wave characteristics for the loading of a slender pile. Proc. 28th Int. Conf. Coastal Eng., ICCE 2002. 1341-1352.

[20] Goring, D. J. And Raichlen, F. 1980 The generation of long waves in the laboratory. Proc. 17th Int. Conf. Coastal Eng., ASCE, New York, 763-783. 
[21] Fenton, J. D. 1988 The numerical solution of steady water wave problems. Computers \& Geosciences, 14 (3), 357-368.

[22] Sobey, R.J. 1989 Variations on Fourier wave theory. International Journal for Numerical Methods in Fluids; 9, 1453-1467. 